\title{
Computational Intelligence in Decision Making
}

\author{
Guest Editors: Tianrui Li, Pawan Lingras, Yuefeng Li, Joseph Herbert
}

This special issue is dedicated to the Fourth International Conference on Rough Sets and Knowledge Technology (RSKT 2009) held in Gold Coast, Australia, July 14-16, 2009. It contains 86 final accepted papers from 229 online submissions for RSKT2009. After peer review, the guess editors accepted 10 revised and extended papers of RSKT2009 to reflect the current development of computational intelligence in decision making.

The first paper by Huaxiong Li and Xianzhong Zhou is entitled "Risk Decision Making Based on Decision-theoretic Rough Set: A Three-way View Decision Model". The authors propose a three-way view decision model based on decision-theoretic rough set model, in which optimistic decision, pessimistic decision, and equable decision are provided according to the cost of misclassification. Then they discuss how to calculate the thresholds of probabilistic inclusion based on minimization of risk cost under respective decision bias. The study provides a practical explanation on diverse risk bias decision.

The second paper by Pawan Lingras and Manish Joshi is entitled "Experimental Comparison of Iterative Versus Evolutionary Crisp and Rough Clustering". The authors compare results obtained using K-means, GA K-means, rough K-means, GA rough K-means and GA rough K-medoid algorithms. They employ a total within cluster variation, average precision, and execution time required as the criteria for comparison. It may contribute to the future applications of algorithms in decision making.

The third paper by Tung-Kuang Wu, Shian-Chang Huang, Ying-Ru Meng, Wen-Yau Liang and Yu-Chi Lin is entitled "Rough Sets as a Knowledge Discovery and Classification Tool for the Diagnosis of Students with Learning Disabilities". The authors introduce the rough set theory to the application of LD diagnosis. Their experiments show that the rough set theory approach is competitive as a tool for feature selection in LD diagnosis, and it performs better in terms of prediction accuracy than other rule-based algorithms.

The fourth paper by Xun Gong, Yong Yang, Jianhui Lin and Tianrui Li is entitled "Expression Detection Based on a Novel Emotion Recognition Method". The authors present a dynamic selection ensemble learning method to analyze human's emotion automatically based on the rough set theory and the domain oriented data driven data mining technique. Experimental results indicate that it is an effective approach for emotion recognition.

The fifth paper by Raymond Y.K. Lau, Chapmann C.L. Lai and Yuefeng Li is entitled "Fuzzy Ontology Mining and Semantic Information Granulation for Effective Information Retrieval Decision Making". The authors illustrate the design and development of a fuzzy ontology based granular information retrieval (IR) system to improve the effectiveness of IR decision making for various domains. A novel computational model is also developed to estimate the semantic granularity of documents by using the fuzzy ontology mining mechanism. Experimental results show that the proposed fuzzy ontology based granular IR system outperforms a classical vector space based IR system in domain specific IR. The study has successfully applied the granular computing and fuzzy ontology mining methods in domain specific IR decision making.

The sixth paper by Dun Liu, Yiyu Yao and Tianrui Li is entitled "Three-way Investment Decisions with Decision-theoretic Rough Sets". The authors adopt the decision-theoretic rough set model to derive a profit-based three-way approach in investment decision-making. A three-way decision is made based on a pair of thresholds on conditional probabilities. The thresholds are calculated by cost 
functions and revenue functions through maximizing conditional profit with the Bayesian decision procedure. The proposed method is validated by a case study in oil investment.

The seventh paper by Dariusz Mrozek and Bożena Małysiak-Mrozek is entitled "An Improved Method for Protein Similarity Searching by Alignment of Fuzzy Energy Signatures". The authors investigate protein comparison by the alignment of protein energy signatures. They represent components of energy signatures in the alignment as fuzzy numbers. It not only improves the decision making while establishing the alignment path, but also guarantees the approximate character of the method.

The eighth paper by Ana Karoline Araújo de Castro, Plácido Rogério Pinheiro, Mirian Calíope Dantas Pinheiro and Isabelle Tamanini is entitled "Towards the Applied Hybrid Model in Decision Making: A Neuropsychological Diagnosis of Alzheimer's Disease Study Case". The authors present a hybrid model, combining influence diagrams and a multicriteria method, to assist with the decision making process in the diagnosis of Alzheimer's disease by considering the stages of Clinical Dementia Rating.

The ninth paper by Yuanxia Shen, Guoyin Wang and Chunmei Tao is entitled "Particle Swarm Optimization with Novel Processing Strategy and Its Application". The authors propose a correlation particle swarm optimization (PSO) model by using a novel correlative strategy to process the personal experience and sharing experience. The study indicates that the processing strategy with positive linear correlation is helpful to maintain the population diversity. Then they propose a positive linear correlation PSO (PLCPSO) where particles adopt the positive linear correlation strategy to process the personal experience and sharing experience. Experimental results show that PLCPSO is an effective optimization approach for complex optimization problems.

The tenth paper by Xiuyi Jia, Lin Shang, Jiajun Chen and Xinyu Dai is entitled "Incremental Versus Non-incremental: Data and Algorithms Based on Ordering Relations". The authors develop an incremental algorithm INRIDDM by means of Dominance-based Rough Set Approach based on multi-dominance discernibility matrices. It is validated that the incremental algorithm INRIDDM is superior to some non-incremental algorithms when dealing with large data sets. They also investigate the influence of data saturation and data concentration on rule induction algorithms. It is concluded that the data saturation and data concentration are crucial for the performance analysis of one learning algorithm.

Finally, the Guest Editors wish to express our deep appreciation to the authors for their contribution, to the reviewers for their careful, insightful and constructive reviews that led to further improvement of the articles. We are thankful to Prof. Da Ruan, Editor-in-Chief of the Journal, for accepting to publish this Special Issue and for his help throughout the publication process.

Tianrui Li

School of Information Science and Technology Southwest Jiaotong University Chengdu 610031, P.R. China Email: trli@swjtu.edu.cn

Pawan Lingras

Department of Mathematics and Computing Science

Saint Mary's University Halifax, Nova Scotia, B3H 3C3, Canada

E-mail: pawan@cs.smu.ca

Yuefeng Li

Faculty of Science and Technology

Queensland University of Technology 
International Journal of Computational Intelligence Systems, Vol.4, No. 1 (February, 2011).

Level 10, 1004, 126 Margaret St, Brisbane, QLD 4001 Email:y2.1i@qut.edu.au

Joseph Herbert

Department of Computer Science

University of Regina

Regina, Saskatchewan

Canada S4S 0A2

Email: herbertj@cs.uregina.ca 


\section{Content}

Huaxiong Li and Xianzhong Zhou, Risk Decision Making Based on Decision-theoretic Rough Set: A Three-way View Decision Model

Pawan Lingras and Manish Joshi, Experimental Comparison of Iterative Versus Evolutionary Crisp and Rough Clustering

Tung-Kuang Wu, Shian-Chang Huang, Ying-Ru Meng, Wen-Yau Liang and Yu-Chi Lin, Rough Sets as a Knowledge Discovery and Classification Tool for the Diagnosis of Students with Learning Disabilities

Xun Gong, Yong Yang, Jianhui Lin and Tianrui Li, Expression Detection Based on a Novel Emotion Recognition Method

Raymond Y.K. Lau, Chapmann C.L. Lai and Yuefeng Li, Fuzzy Ontology Mining and Semantic Information Granulation for Effective Information Retrieval Decision Making

Dun Liu, Yiyu Yao and Tianrui Li, Three-way Investment Decisions with Decision-theoretic Rough Sets

Dariusz Mrozek and Bożena Małysiak-Mrozek, An Improved Method for Protein Similarity Searching by Alignment of Fuzzy Energy Signatures

Ana Karoline Araújo de Castro, Plácido Rogério Pinheiro, Mirian Calíope Dantas Pinheiro and Isabelle Tamanini, Towards the Applied Hybrid Model in Decision Making: A Neuropsychological Diagnosis of Alzheimer's Disease Study Case

Yuanxia Shen, Guoyin Wang and Chunmei Tao, Particle Swarm Optimization with Novel Processing Strategy and Its Application

Xiuyi Jia, Lin Shang, Jiajun Chen and Xinyu Dai, Incremental Versus Non-incremental: Data and Algorithms Based on Ordering Relations 